\title{
Tripartite Entanglement for Qubits and Qudit in Double Photoionization of Xenon Atom
}

\author{
M. Chakraborty ${ }^{1}$ and S.Sen ${ }^{2}$ \\ ${ }^{1}$ Department of Physics, Asansol Girls' College, Asansol -713304, India \\ ${ }^{2}$ Department of Physics; Triveni devi Bhalotia College, Raniganj- 713347, India \\ bminakshi@yahoo.com; sandip_sn@yahoo.com
}

\begin{abstract}
Quantum entanglement holds the key to an information processing revolution. In this article, we study the entanglement properties of tripartite states of two electronic qubits and ionic qudit, without observing spin orbit interaction (SOI), produced by single-step double photoionization from Xeon atom following the absorption of a single photon. The dimension of the Hilbert space of the qudit depends upon the electronic state of the residual photoion $\mathrm{Xe}^{2+}$. In absence of SOI, Russel-Salunders coupling (L-S coupling) is applicable. As the estimations of entanglement, we consider Peres-Horodecki condition and negativity. In case of L-S coupling, all the properties of a qubit-qudit system can be predicted merely with the knowledge of the spins of the target atom, the residual photoion, emitted electrons and state of polarization of the incident photons.
\end{abstract}

Keywords - entanglement, quantum information, qubit, qudit, Peres-Horodecki condition, negativity

\section{Introduction}

Quantum entanglement is a key prediction of quantum mechanics and one of the resources needed in quantum information (QI) processing [1, 2]. In analogy to the classical information, QI also needs bits, called qubits. But, a bit in QI is a quantum system which has, at least, one observable requiring two-, or higher-, dimensional space for its characterization. A two-dimensional quantum system (e.g., a spin- $\frac{1}{2}$ particle) is called in QI as a quantum bit or qubit [3,4].In general, a d-dimensional quantum system (with $\mathrm{d}>2$ ) is called a qudit [5].

Availability of two or more qubits with entanglement is an essential ingredient for any quantum information related studies. Quantum entanglement is a nonlocal property that allows a set of qubit to express higher correlation that is not possible in classical systems.

Research into quantum entanglement was started in 1935 by A. Einstein, B. Polodolsky and N. Rosen describing the EPR paradox [6]. The flaw in EPR arguments was discovered by J. S. Bell [7], who proved that the principle of locality used in EPR paradox, was not consistent with the hidden variables interpretation of quantum theory. Bell's theorem conversely provides one of the possible methods to test whether two or more particles form an entangled state. The strong correlations among entangled 
M. Chakraborty and S.Sen; Tripartite Entanglement for Qubits and Qudit in Double Photoionization of Xenon Atom, Transactions on Machine Learning and Artificial Intelligence, Volume 7 No 1 February, (2019); pp: 1-9

particles can be used as resources of quantum cryptography [8], quantum teleportation [9] and quantum computation [10].

It is known that quantum correlation in qudit or multipartite system is stronger than in the bipartite system. The investigations performed hitherto have already shown that entanglement among more than two particles is not merely an extension of its bipartite counterpart but has instead several new and different properties which are more advantageous, as example, in super dense coding, quantum cloning, teleportation [11]. Koike el al. [12] have already demonstrated experimentally " $\rightarrow 2$ quantum telecloning" for optical coherent states. To study QI at more fundamental levels, the multipartile entangled states, in addition, are also needed. It has been suggested [13] that the use of qudit can reduce the number of qubits by a function of $\log _{2} d$.

In section 2, we briefly describe the density operator (DO) and states for DPI of an atom. This operator corresponds to the case when the ionizing electromagnetic radiation is in a pure state of polarization and the target atom is in its ground state before DPI. In section 3, we study the entanglement in DPI for qubits and qudit system. A quantitative application of entangled properties in DPI of xenon is presented in section 4 . Finally section $\mathbf{5}$ contains the conclusion part.

\section{Preliminaries}

\subsection{Density Operators:}

Let us represent by $e_{1}$ and $e_{2}$ the two freely moving electrons whose entanglement properties we want to investigate. The propagation vector of the $i(=1,2)$-th electron is $\vec{k}_{i}=\left(k_{i}, \theta_{i}, \phi_{i}\right)$ such that its kinetic energy is given by $\varepsilon_{i}=\hbar^{2} k_{i}^{2} / 2 m$. Also, $\mu_{i}(= \pm 1 / 2)$ represents the projection of the spin angular momentum of $i$-th electron along its spin quantization direction $\hat{u}_{i}=\left(\alpha_{i}, \beta_{i}\right)$. These two electrons form an integral part of an atom $A$ are assumed to be simultaneously ejected from it following the absorption of a single photon. If $\mathrm{A}^{2+}$ denotes the residual dication, then our process can schematically be represented by

$$
h v_{r}\left(\left|r_{r}\right|=1, m_{r}\right)+A|0\rangle \rightarrow A^{2+}|f\rangle+e_{1}\left(\vec{k}_{1} ; \mu_{1} \hat{u}_{1}\right)+e_{2}\left(\vec{k}_{2} ; \mu_{2} \hat{u}_{2}\right) .
$$

Here $E_{r}=h v_{r}$ andb $\left|l_{r}\right|=1$ are, respectively, the energy and the angular momentum of the absorbed photon. Let us denote by $\rho_{0}=|0\rangle\langle 0|$ and $\rho_{r}=\left|m_{r}\right\rangle\left\langle m_{r}\right|$ the respective density operators of the unpolarized atom $A$ before DPI and of the ionizing radiation. This means the density operator of the combined (atom + photon) system of Eq. (1) is given by the direct product

$$
\rho_{i}=\rho_{0} \otimes \rho_{r}
$$




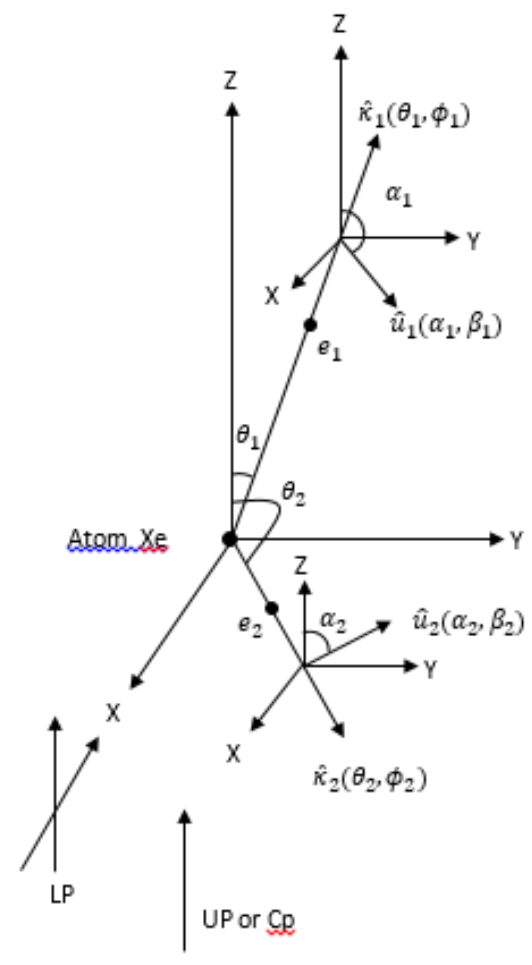

Figure 1. Two electrons are emitted simultaneously after photoabsorption.

Let us denote by $F_{p}$ the photoionization operator in the $E 1$ approximation. Then the density operator of the combined $\left(A^{2+}+e_{1}+e_{2}\right)$ system in equation (1) after DPI becomes

$$
\rho_{f}=K_{p} F_{p} \rho_{i} F_{p}^{+}
$$

Here, $K_{p}=3 \pi\left(e^{2} / \alpha_{0} E_{r}\right)^{2}$ with $\alpha_{0}$ the dimensionless fine structure constant [14].

\subsection{Criteria and measures for entanglement and mixedness of a state}

Only recently, quantum entanglement is recognized as resources with various applications such as quantum teleportation and quantum key distribution in the field of quantum information and quantum computation. So, the task of characterizing and quantifying entanglement has emerged as one of the prominent challenge of quantum information theory. The quantum entanglement for multipartite states poses even a greater challenge. The condition for separability given by Peres [15] and Horodecki family [16] is that the partial transpose (PT), with respect to either of the two particles, of its DM remains positive. We have applied Peres-Horodecki positive partial transpose (PPT) condition in order to characterization of entanglement. As a measure of the degree of entanglement we consider negativity [4, 17]

As a measure we consider the negativity, which is an additive and operational measure of entanglement. Additivity is a very desirable property that can reduce computation of entanglement. It can also be generalized to multipartite and higher dimensional entanglement. The negativity of a state [18] indicates to which extent a state violates the positive partial transpose separability criterion. The negativity is twice [16] the absolute value of the sum of negative eigenvalues: 


$$
N=2 \max \left(0,-\lambda_{\text {neg }}\right)
$$

where $\lambda_{\text {neg }}$ is the sum of the negative eigenvalues of $\rho_{A B}^{T_{B}}$, is the partial transpose of $\rho_{A B}$.

For higher dimensional mixed states, the negativity [19], however, can be defined as

$$
N=\frac{\left\|\rho_{A B}^{T_{B}}\right\|_{1}-1}{d-1}
$$

for the states in a $d \otimes d^{\prime}\left(d \leq d^{\prime}\right)$ quantized system and \|\|$_{1}$ is the trace norm.

\section{Entanglement between Two Electronic Qubits and an Ionic Qudit for DPI}

\subsection{Density matrix}

Here, we calculate the DM for the angle- and spin-resolved DPI of an atom without considering SOI (L-S coupling) into account in either of the bound electronic states of $A$ and $A^{2+}$ or in the continua of the two photoelectrons $\left(e_{1}, e_{2}\right)$ ejected in the process (1). As LS-coupling is applicable here, the total orbital angular momenta $\left(\vec{L}_{0}, \vec{L}_{f}\right)$ and the spin angular momenta $\quad\left(\vec{S}_{0}, \vec{S}_{f}\right)$ of $A$ and $A^{2+}$ are the conserved quantities. If, the orbital angular momentum of the photoelectron $e_{1}$ is $\vec{l}_{1}$ and that of $e_{2}$ is $\vec{l}_{2}$ with their spin angular momenta $\left(\frac{1}{2}\right)_{1}$ and $\left(\frac{1}{2}\right)_{2}$ respectively, we then have

$$
\vec{L}_{0}+\vec{l}_{r}=\vec{L}_{f}+\vec{l}\left(=\vec{l}_{1}+\vec{l}_{2}\right)
$$

and

$$
\vec{S}_{0}=\vec{S}_{f}+\vec{S}_{t}\left[=\left(\frac{1}{2}\right)_{1}+\left(\frac{1}{2}\right)_{2}\right] .
$$

Here we use the symbols $M_{L_{0}}, M_{S_{0}}, M_{L_{f}}$ and $M_{S_{f}}$ to represent the respective projections of $\vec{L}_{0}, \vec{S}_{0}, \vec{L}_{f}$ and $\vec{S}_{f}$ along the polar axis of the space frame. In equation (1), the bound electronic state of atom $A$ is $|0\rangle \equiv\left|L_{0} S_{0} M_{L_{0}} M_{S_{0}}\right\rangle$ and that of the dication $A^{2+}$ is $|f\rangle \equiv\left|L_{f} S_{f} M_{L_{f}} M_{S_{f}}\right\rangle$. The density operator (2) for the combined (atom + photon) system can be written as

$$
\rho_{i}=\frac{1}{\left(2 L_{0}+1\right)\left(2 S_{0}+1\right)} \sum_{M_{L_{0}} M_{S_{0}}}\left|0 ; 1 m_{r}\right\rangle\left\langle 0 ; 1 m_{r}\right|,
$$

where we have defined $\left|0 ; 1 m_{r}\right\rangle \equiv|0\rangle\left|1 m_{r}\right\rangle$.

In order to calculate the DM for the $\left(\mathrm{A}^{2+}+\mathrm{e}_{1}+\mathrm{e}_{2}\right)$ system in process (1), we need to calculate the matrix elements $\rho_{i}$ and $\rho_{f}$. Following the procedures given in reference [20] the matrix elements in the present case are given by 


$$
\begin{gathered}
\left\langle f ; \vec{k}_{1}, \mu_{1} \hat{u}_{1}, \vec{k}_{2}, \mu_{2} \hat{u}_{2}\left|\rho_{f}\right| f ; \vec{k}_{1}, \mu_{1}^{\prime} \hat{u}_{1}, \vec{k}_{2}, \mu_{2}^{\prime} \hat{u}_{2}\right\rangle=\frac{K_{p}}{\left(2 L_{0}+1\right)\left(2 S_{0}+1\right)} \sum_{M_{L_{0}} M_{S_{0}}}\left\langle f ; \vec{k}_{1}, \mu_{1} \hat{u}_{1} ; \vec{k}_{2}, \mu_{2} \hat{u}_{2}\left|F_{p}\right| 0 ; 1 \mathrm{~m}_{r}\right\rangle \\
\mathrm{x}\left\langle f ; \vec{k}_{1}, \mu_{1}^{\prime} \hat{u}_{1}, \vec{k}_{2}, \mu_{2}^{\prime} \hat{u}_{2}\left|F_{p}\right| 0 ; 1 \mathrm{~m}_{r}\right\rangle^{*}
\end{gathered}
$$

The DM of the tripartite system for angle- and spin-resolved DPI process (1) in the absence of SOI can be written in the following form:

$$
\left\langle f ; \vec{k}_{1}, \mu_{1} \hat{u}_{1} ; \vec{k}_{2}, \mu_{2} \hat{u}_{2}\left|\rho_{f}\right| f ; \vec{k}_{1}, \mu_{1}^{\prime} \hat{u}_{1} ; \vec{k}_{2}, \mu^{\prime}{ }_{2} \hat{u}_{2}\right\rangle=\frac{d^{3} \sigma\left(m_{r}\right)}{d \varepsilon_{1} d \hat{k}_{1} d \hat{k}_{2}} \times \sigma\left(S_{0} ; S_{f} ; \hat{u}_{1}, \hat{u}_{2}\right)_{M_{S_{f}} \mu_{1} \mu_{2} ; M_{S_{f}}^{\prime} \mu_{1}^{\prime} \mu_{2}^{\prime}} .
$$

The first term i.e., the triple differential cross section (TDCS i.e., $\left.d^{3} \sigma\left(m_{r}\right) / d \varepsilon_{1} d \hat{k}_{1} d \hat{k}_{2}\right)$ on the right-hand side of (7) depends upon the orbital angular momenta of $A$ and $A^{2+}$, phase shifts, energies $\left(\varepsilon_{1}, \varepsilon_{2}\right)$ and the directions $\left(\hat{k}_{1}, \hat{k}_{2}\right)$ of the emitted electrons $\left(e_{1}, e_{2}\right)$, the state of polarization $\left(m_{r}\right)$ of the ionizing radiation and the photoionization dynamics. It does not include spins of the photoelectrons or the target atom or the residual dication. Thus, the TDCS $d^{3} \sigma\left(m_{r}\right) / d \varepsilon_{1} d \hat{k}_{1} d \hat{k}_{2}$ in the DM (7) describes angular correlation between the photoelectrons and the residual ion. Its value is always positive. Here,

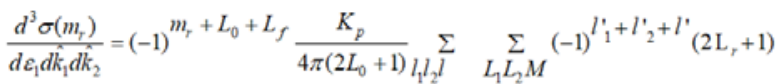

$$
\begin{aligned}
& l_{1}^{\prime} l_{2}^{\prime} l^{\prime} L L^{\prime} L_{r}
\end{aligned}
$$

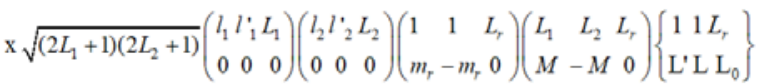

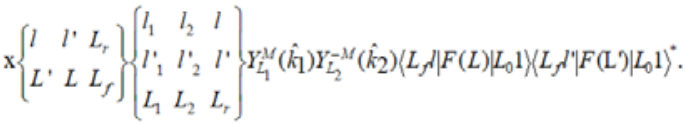

The second term (i.e., $\left.\sigma\left(S_{0} ; S_{f} ; \hat{u}_{1}, \hat{u}_{2}\right)_{M_{f} \mu_{1} \mu_{2} ; M^{\prime}{ }_{f} \mu_{1}^{\prime} \mu_{2}^{\prime}}\right)$ is the spin-correlation matrix. It completely determines the properties of the Coulombic entanglement among $\left(e_{1}, e_{2}\right.$ and $\left.A^{2+}\right)$. It can be written as

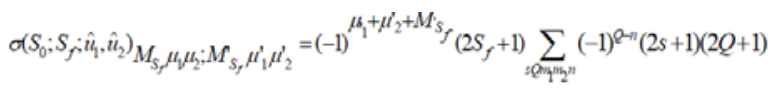

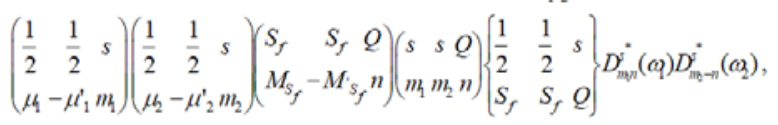

where $D$ s are the rotational harmonics [21] with $\omega_{1}\left(\alpha_{1}, \beta_{1}, 0\right)$ and $\omega_{2}\left(\alpha_{2}, \beta_{2}, 0\right)$ the Euler angles which rotate the axis of the space-frame into the spin-polarization directions $\hat{u}_{1}$ and $\hat{u}_{2}$ (Fig. 1 ), respectively.

In expression (9) the allowed values for each of $\mu_{1}, \mu_{1}^{\prime}, \mu_{2}$ and $\mu_{2}^{\prime}$ are $\pm \frac{1}{2}$, the spin magnetic quantum numbers $M_{S_{f}}$ and $M^{\prime}{ }_{S_{f}}$ of the photoion $A^{2+}$ can take $\left(2 S_{f}+1\right)$ values. So, DM (9) of size $\left[2\left(2 S_{f}+1\right) \times 2\left(2 S_{f}+1\right)\right]$ represents spin correlations between the electronic qubits $\left(e_{1}, e_{2}\right)$ and $\mathrm{d}[=$ $\left.\left(2 S_{f}+1\right)\right]$-dimensional ionic qudit $A^{2+}$. Hence, the dimensionality $d$ of the qudit can be chosen according to the appropriate spins $S_{0}$ and $S_{f}$ of the atomic target $A$ and photoion $A^{2+}$, respectively.

The $d^{3} \sigma\left(m_{r}\right) / d \varepsilon_{1} d \hat{k}_{1} d \hat{k}_{2}$ term in (7) represents the angular correlation of two qubits-qudit system and always has a positive value. Therefore, unless stated otherwise, we write the DM (7) as 


$$
\left\langle f ; \vec{k}_{1}, \mu_{1} \hat{u}_{1} ; \vec{k}_{2}, \mu_{2} \hat{u}_{2}\left|\rho_{f}\right| f ; \vec{k}_{1}, \mu_{1}^{\prime} \hat{u}_{1} ; \vec{k}_{2}, \mu_{2}^{\prime} \hat{u}_{2}\right\rangle=\sigma\left(S_{0} ; S_{f} ; \hat{u}_{1}, \hat{u}_{2}\right)_{M_{S_{f}} \mu_{1} \mu_{2} ; M_{S_{f}} \mu_{1}^{\prime} \mu_{2}^{\prime}}
$$

In order to study the entanglement properties for DPI in (1), we need to calculate partial transpose (PT) $[14,15]$ of the $\operatorname{DM}(10)$ with respect to either of three subsystems, i.e., photoelectrons $\left(e_{1}, e_{2}\right)$ and photoion $A^{2+}$. These are given by

$$
\begin{gathered}
\sigma^{{ }^{A^{2+}}}=\sigma\left(S_{0} ; S_{f} ; \hat{u}_{1}, \hat{u}_{2}\right)_{M_{S_{f}}^{\prime} \mu_{1} \mu_{2} ; M_{S_{f}} \mu_{1}^{\prime} \mu_{2}^{\prime}}, \\
\sigma^{T_{e_{1}}}=\sigma\left(S_{0} ; S_{f} ; \hat{u}_{1}, \hat{u}_{2}\right)_{M_{S_{f}} \mu_{1}^{\prime} \mu_{2} ; M_{S_{f}}^{\prime} \mu_{1} \mu_{2}^{\prime}}
\end{gathered}
$$

and

$$
\sigma^{T_{2}}=\sigma\left(S_{0} ; S_{f} ; \hat{u}_{1}, \hat{u}_{2}\right)_{M_{S_{f}} \mu_{1} \mu_{2}^{\prime} ; M^{\prime} S_{f} \mu_{1}^{\prime} \mu_{2}}
$$

\subsection{Tripartite Entanglement}

We have also studied tripartite entanglement properties between ionic qudit and electronic qubit system. The tripartite entanglement can be converted into two stand forms [4.11] namely $|\mathrm{GHZ}\rangle$ and $|\mathrm{W}\rangle$ states. We use $|\mathrm{W}\rangle$ state as it is more suitable for mixed state entanglement and can be written as

$$
|\mathrm{W}\rangle=\frac{1}{\sqrt{3}}(|001\rangle+|010\rangle+|100\rangle)
$$

We calculate tripartite entanglement using equations (10), (11) and (12) for the geometry where each of the photo electrons is polarized in X-Y plane $\left(\beta_{1}\right.$ and $\left.\beta_{2}=\pi / 2\right)$ of the space frame.

(i) We have first studied the case when $\left[S_{0}=S_{f}-1\right]$ with $S_{f} \geq 1$. Without specifying the spin $S_{f}$ we are not able to diagonalize the DM. We have calculated the DM (10) and its partial transpose using equation (11) for each of the $S_{f}=\left(1, \frac{3}{2}, 2, \frac{5}{2}, 3\right)$. For quantifying entanglement we have used negativity as

$$
N\left[\sigma\left(S_{0}=S_{f}-1 ; S_{f} \geq 1\right)\right]=\frac{\sqrt{6}}{2 S_{f}+1} \text { with } S_{f} \geq 1 .
$$

We can see from Eq. (13) that the tripartite system is partially entangled for all values of $S_{f} \geq 1$ and the value of negativity ranges from 0 to $\sqrt{2 / 3}$, decreasing with the increasing value $S_{f}$, the spin of ionic qudit $A^{2+}$.

(ii) Next we study the case for $S_{0}=S_{f}+1$ with $S_{f} \geq 1$. We have calculated the DM (10) and its partial transpose using equation (11) for each value of the $S_{f}=\left(1, \frac{3}{2}, 2, \frac{5}{2}, 3\right)$. In this case we have seen that none of the eigenvalues of the partial transpose (11) is negative. So, the states for 
$\left[S_{0}=S_{f}+1\right]$ with $S_{f} \geq 1$ have positive partial transpose (PPT). So, the states for $\left[S_{0}=S_{f}+1\right]$ with $S_{f} \geq 1$ may possess bound entanglement if $4\left(2 S_{f}+1\right)>6$, i.e. $S_{f} \geq 1$ and obeys the inequality $[4,13]$

$$
\operatorname{Rank}\left[\sigma\left(S_{0}=S_{f}+1 ; S_{f} \geq 1\right)\right] \geq \operatorname{Max}\left\{\operatorname{Rank}\left[\sigma\left(e_{1}\right)\right], \operatorname{Rank}\left[\sigma\left(e_{2}\right)\right], \operatorname{Rank}\left[\sigma\left(A^{+2}\right)\right]\right\}
$$

In this case, $\operatorname{Rank}\left[\sigma\left(S_{0}=S_{f}+1 ; S_{f} \geq 1\right)\right]$ is $4\left(2 S_{f}+1\right), \quad \operatorname{Rank}\left[\sigma\left(e_{1}\right)\right]$ is $2, \operatorname{Rank}\left[\sigma\left(e_{2}\right)\right]$ is 2 and $\operatorname{Rank}\left[\sigma\left(A^{+2}\right)\right]$ is $\left(2 S_{f}+1\right)$. So, the states for $\left[S_{0}=S_{f}+1\right]$ with $S_{f} \geq 1$ obey the condition (14) and hence possess bound entanglement.

\section{Example for Tripartite Entanglement In DPI For Xenon}

As an application for the case $\left|S_{f}-S_{0}\right|=0$ in in xenon atom. The DPI process in the ground electronic state of xenon atom is the following:

$$
h v_{r}+X e\left(4 d^{10} 5 s^{2} 5 p^{63} P_{0}\right) \rightarrow X e^{2+}\left(4 d^{9} 5 s^{1} 5 p^{61} P_{0}\right)+e_{1}\left(\vec{k}_{1} ; \mu_{1} \hat{u}_{1}\right)+e_{2}\left(\vec{k}_{2} ; \mu_{2} \hat{u}_{2}\right) .
$$

We use the values of TDCS of Xenon given in Ref. [22] for photon energy $1 \mathrm{eV}$ for the geometry $\theta_{12}=180^{\circ}$ ,where $\theta_{12}$ is the angle between two ejected electrons. The TDCS are

$$
\left.\frac{d^{3} \sigma\left(\theta_{12}=180^{\circ}\right)}{d \varepsilon_{1} d \hat{k}_{1} d \hat{k}_{2}}\right|_{p}=4 a^{2} \mathrm{E}^{n-\frac{3}{2}} \sin ^{2} \theta_{1}
$$

And

$$
\left.\frac{d^{3} \sigma\left(\theta_{12}=180^{\circ}\right)}{d \varepsilon_{1} d \hat{k}_{1} d \hat{k}_{2}}\right|_{u}=4 a^{2} \mathrm{E}^{n-\frac{3}{2}}\left(1+\cos ^{2} \theta_{1}\right)
$$

for polarized and unpolarized photons respectively. The variation of Negativity entropy with respect to the direction of ejection and spin polarization of the photoelectrons is shown below

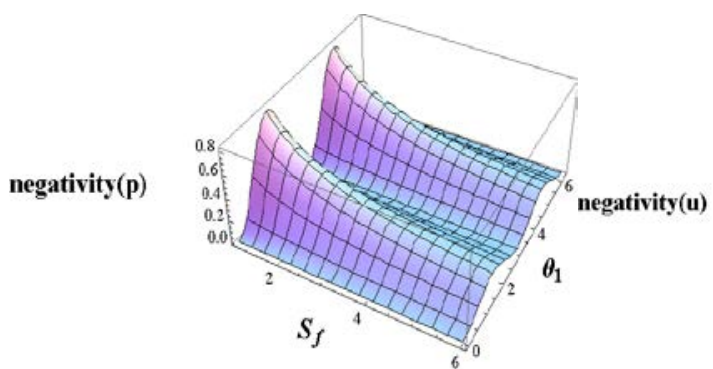

(a)

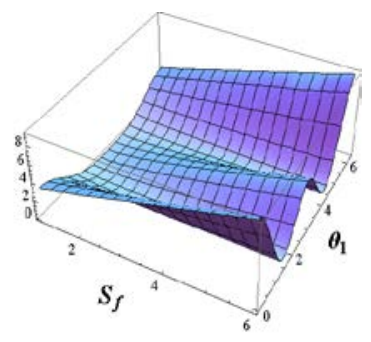

(b)

Figure 2. Variation of Negativity with respect to $S_{f}$ and $\boldsymbol{\theta}_{f}$ 
M. Chakraborty and S.Sen; Tripartite Entanglement for Qubits and Qudit in Double Photoionization of Xenon Atom, Transactions on Machine Learning and Artificial Intelligence, Volume 7 No 1 February, (2019); pp: 1-9

From figure 2 we see that depending on direction of ejection of photoelectrons and state of polarization of incident photons for $\left|S_{f}-S_{0}\right|=0$ in xenon atom are partially entangled and unentangled and magnitude of negativity is also effected by TDCS.

\section{Conclusion}

Entanglement between a pair of particles is a very delicate thing and is easily destroyed. Currently, many scientists are working on making stronger systems where entanglement is stronger and lasts longer to use for quantum computation and information more easily. Photons are, probably the fastest and best carriers of information but they are not suitable for storing information for a long period of time [23]. lons (positive or negative) and electrons are stable, easily detectable and capable of storing information for a long time. Electrons are the lightest particles next to photon therefore able to travel with speeds comparable with light. Furthermore, electrons have since long been used to carry information in the form of electric signals and /or for storing it for a long time. It was already suggested [24] that electron spin can be used for quantum computation [1].

DPI is the most natural processes for simultaneously producing two electrons in continuum in a single step. It is the most direct manifestation of electron- electron correlation in an atom, because had the independent particle model been valid, two electrons would not have emerged simultaneously following the absorption of a single photon. Thus, simultaneous ejection of two electrons from an atom, after the absorption of a single photon, could take place due to the existence of correlation effects between them. Here we have tried to show that the DPI process is a powerful tool for investigations of tripartite entanglement between two electronic qubits and an ionic qudit. The entanglement is quantified by PeresHorodecki's NPT condition and negativity. These bipartite and tripartite systems may be pure or mixed and may possess free or bound entanglement[25]. They may be totally entangled, partially entangled or separable depending on spin states of target $A$ and residual dication $A^{2+}$ as well as of the directions spin quantization and ejection of the photoelectrons [26].

A quantitative case is studied for DPI in Xenon atom and has been shown that the TDCS effects

entanglement. For Xenon atom we have studied the states for $\left|S_{f}-S_{0}\right|=0$ and have shown that depending on the direction of ejection of photoelectrons and state of polarizations, the states are totally entangled, partially entangled and unentangled.

\section{REFERENCES}

[1] Quantum Computation and Quantum Information, M.A. Nielsen and I. L. Chuang (Cambridge University Press, Cambridge, England, 2000).

[2] Quantum Information: An Introduction to basic Theoretical Concepts and Experiments, edited by G. Alber, T. Berth, M. Horodecki, P. Horodecki, R. Horodecki, M. Rötteler, H. Weinfurter, R. Werner and A. Zeilinger (Spinger, Berlin, 2000) .

[3] B. Shumacher, Phys. Rev. A 51, 2738 (1995).

[4] M. Horodecki, P. Horodecki and R. Horodecki, Rev. Mod. Phys. 81, 865 (2009). 
[5] P. Rungta et al., in Directions in Quantum Optics: A Collection of Papers Dedicated to the Memory of Dan Walls, edited by H. J. Carmichae et al., (Spinger, Berlin, 2001), pp. 149-164.

[6] A. Einstein, B. Polodolsky and N. Rosen, Phy. Rev. 47, 777 (1935).

[7] J. S. Bell, Physics 1, 195 (1965).

[8] A. Ekert, Phys. Rev. Lett. 67, 661 (1991).

[9] C. H. Bannett, C. Brassard, R. Jozsa, A. Peres and W. K. Wooters, Phys. Rev. Lett. 70, 1895 (1993).

[10] A. Ekert and. R. Jozsa, Rev. Mod. Phys. 68, 733 (1998).

[11] S. Parida and N. Chandra, Phys. Rev. A 86, 062302 (2012).

[12] S. Koike, H. Takahashi, H. Yonezawa, N. Takei, S. L. Braunstein, T. Aoki and A. Furusawa, Phys. Rev. Lett. 96, 060504 (2006).

[13] A. Galindo and M.A. Martin-Delgado, Rev. Mod. Phys.74, 347 (2002).

[14] C.H. Bennett, D. P. DiVincenzo, J. Smolin, and W. K. Wootters, Phys. Rev. A 54, 3824 (1996).

[15] A. Peres, Phys. Rev. Lett. 77, 1413 (1996).

[16] M. Horodecki, P. Horodecki and R. Horodecki, Phys. Rev. A. 223, 1, (1996).

[17] T.C. Wei, K. Nemoto, P. M. Goldbart, P. G. Kwiat, W. J. Munro and F. Verstraete, Phys. Rev. A 67, 022110 (2003).

[18] S. Ishizaka and T. Hiroshima, Phys. Rev. A 62, 022310 (2000).

[19] W. K. Wootters, Phys. Rev. Lett. 80, 2245 (1998).

[20] N. Chandra and M. Chakraborty, J. Phys. B: At. Mol. Opt. Phys. 35, 2219 (2002).

[21] Angular Momentum in Quantum Mechanics, A. R. Edmonds (Princeton University press, Princeton, 1974).

[22] A Huetz, P. Selles, D. Waymel and J. Mazeau, J. Phys. B 24 ,1917 (1991).

[23] Quantum Entanglement in Electron Optics, N. Chandra and R. Ghosh, (Springer-Verlag, Berlin, 2013).

[24] D. Loss, D. P. DiVicenzo, Phys. Rev. A 57, 120 (1998).

[25] M. Chakraborty and S. Sen, Journal of Electron Spectroscopy and Related Phenomena,203, 60 (2015).

[26] M. Chakraborty, Indian Journal of Science and Technology, 10, 27 (2017). 